\section{Fighting cancer with microbes}

\section{Targeting the microbiome could hold the key to combating a range of malignant diseases. By Elie Dolgin}

t n July 1984, a young Australian gastroenterologist drank a beef broth spiked with the pathogenic bacterium Helicobacter pylori. Within a week, he started vomiting. His breath began to stink. And he couldn't have been happier.

Barry Marshall wanted to prove that $H$.pylori could trigger inflammation of the stomach lining, a first sign of stomach cancer. By taking a biopsy of his own stomach tissue, Marshall demonstrated unequivocally that the hardy, spiral-shaped microorganism could cause gastric disease. Twenty-one years later, Marshall and his mentor Robin Warren won the Nobel Prize in Physiology or Medicine for their discovery linking the bacterium to chronic inflammation, peptic ulcers and stomach ailments such as cancer.

Yet $H$. pylori was long considered to belong to a special club of infectious agents, together with viruses such as human papillomavirus, that could provoke tumour formation. In oncology circles, the trillions of microbes that inhabit our guts, skin and other tissues were seen mostly as benign bystanders.

Cancer researchers now realize that many of those seemingly harmless microbes are anything but. Over the past decade, it's become clear that gut microbes can produce DNA-damaging toxins and carcinogenic metabolites, induce cancer-promoting inflammation, make tumours more resistant to chemotherapy drugs, and suppress the body's anticancer immune responses. "Every day now there seems to be some new microbe associated with cancer," says Susan Bullman, a microbiologist at the Fred Hutchinson Cancer Research Center in Seattle, Washington.

While researchers such as Bullman are now racing to unravel the molecular mechanisms behind tumour-promoting bacteria - and in so doing, identify targets for risk assessment, early detection, prevention and treatment many cancer researchers are already testing whether microbiome-based therapeutics can improve the efficacy or safety of existing anti-cancer interventions. Borrowing a page from the gastroenterology playbook, oncologists around the world have begun giving their patients faecal transplants and bacteria-filled capsules.

These living medicines have already revolutionized the treatment of antibiotic-resistant gut infections. A few studies have also shown the potential of faecal transplants for people with blood cancers receiving a stem-cell transplant. (These patients must take broad-spectrum antibiotics to prevent infections, but in so doing they lose the bacteria that are needed to prevent donated cells attacking the host.) Now, researchers are beginning to find that a dose of beneficial microbes enhances the efficacy of immune-modulating drugs known as checkpoint inhibitors and mitigates toxicity.

"Modulating the microbiome makes complete sense," says Jennifer Wargo, a surgical oncologist at the University of Texas MD Anderson Cancer Center in Houston. "People are really embracing the idea and we're beginning to see the early fruits of that labour."

\section{Not the only bad guy}

Microbiologist Jun Yu of the Chinese University of Hong Kong has begun to take a close look at the role that bacteria have in driving stomach cancer. Yu's team identified a handful of microbes that were consistently enriched in samples from people with gastric cancer ${ }^{1}$ or precancerous stomach lesions. "H. pylori is not the only bad guy," she says.

Yu suspects that the focus on $H$. pylori was an accident of history. This microbe happened to grow in laboratory cultures - the standard technique for bacterial identification during the 1980s. Yu's team relied on DNA analyses instead. "Gene sequencing provides a good opportunity to identify other microbes in the stomach that also play some role but weren't discovered before," she says. Her team is now evaluating the tumour-causing potential of these bacteria in mouse models.

Gut microbes have also been linked to bowel cancer, the third most common type of cancer worldwide. A toxin produced by a strain of gut

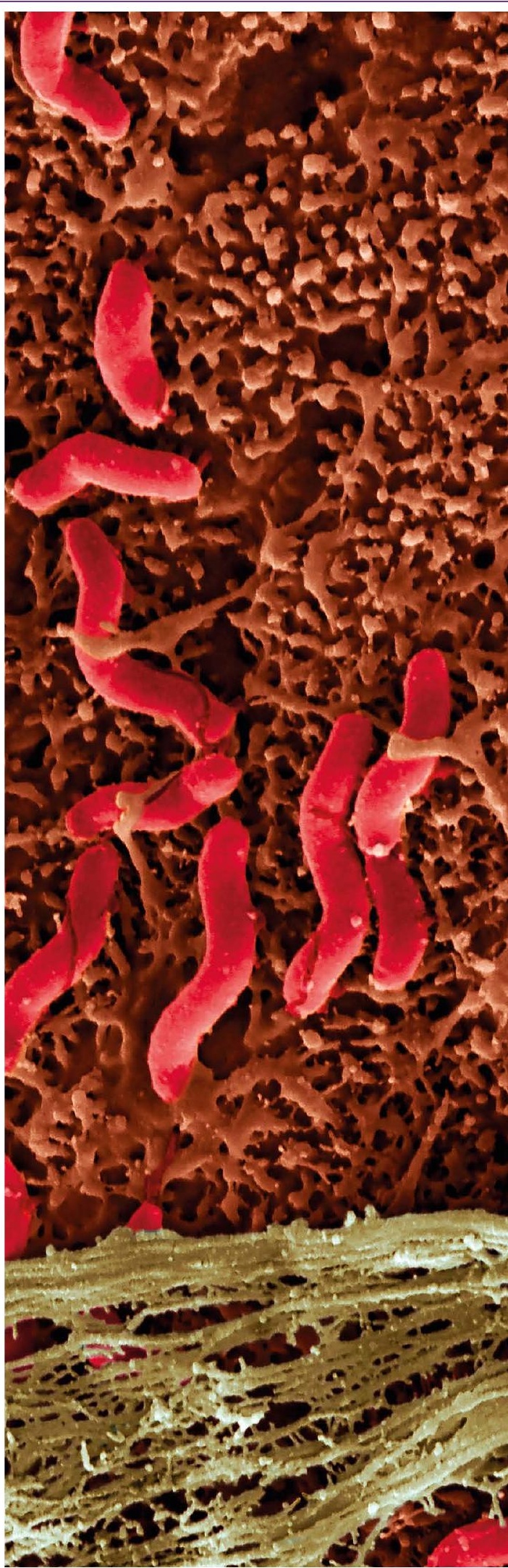

Scanning electron micrograph of Helicobacter pylori on the surface of the intestine. 
bacterium called Bacteroides fragilis, a cause of diarrhoeal disease in young children, was implicated in the development of bowel cancer in 2009. Cynthia Sears, an infectious-disease specialist at Johns Hopkins University in Baltimore, Maryland, who made the original discovery in mice, has since shown that the toxin recruits immune cells to the intestinal lining and promotes an inflammatory cascade that can lead to cancer².

And a team led by Christian Jobin, an immuno-microbiologist at the University of Florida in Gainesville, made a similar discovery: a strain of Escherichia coli that induces bowel cancer through the production of a toxin that damages DNA ${ }^{3}$. None of these pathogens necessarily work in isolation, however. "It's an assembly of microorganisms that can collectively impact genome stability and immune function," Jobin says.

Evidence for the idea that both $B$. fragilis and E. coli work together to fuel tumour growth comes from Sears, who showed that people who are genetically predisposed to bowel cancer frequently have patchy bacterial biofilms in their colons composed predominantly of these two microbes ${ }^{4}$.

\section{Menace of microbes}

Although it did not crop up in Sears's study, there are multiple lines of evidence that the bacterium Fusobacterium nucleatum - a spindle-shaped microbe found in most people's mouths - is also a major driver of bowel cancer. It seems to promote proliferation of cancer cells through direct interactions with intestinal cells, rather than through a toxin intermediary.

The association between $F$. nucleatum and cancer first emerged through sequencing-based studies of tumour samples. Immunogeneticist Robert Holt at the British Columbia Cancer Agency in Vancouver, Canada, compared RNA from 11 bowel tumours with sequences from adjacent normal tissues ${ }^{5}$. When he and his colleagues looked for microbial genes expressed at elevated levels in the tumours, "the Fusobacterium signal really jumped out", Holt says. Around the same time, a team led by immunologist Wendy Garrett and cancer geneticist Matthew Meyerson at Dana-Farber Cancer Institute in Boston, Massachusetts - stumbled on the same microbe after analysing the DNA of bowel tumours ${ }^{6}$.

In the years since, research teams from the Czech Republic, China, Japan and South Korea have all found that people with higher levels of $F$. nucleatum in their bowel tumours tend to have worse survival outcomes. The biological explanation, however, remains elusive. "If you have Fusobacterium and advanced colorectal cancer, that sucks because your time to progression is going to be shorter," Garrett says. "But why is that?"

Research in mice by scientists including Garret suggests that the presence of $F$. nucleatum increases cancer growth, possibly through the modulation of immune responses or through the direct activation of cancer signalling pathways. The microbe also seems to promote resistance to chemotherapy through the induction of a cellular recycling process that enhances tumour survival in the face of drugs. Other tumour-resident bacteria can metabolize chemotherapeutics, which further contributes to drug resistance.

Last year, an international team led by Garrett and Meyerson secured a $£ 20$-million (US\$26-million) grant from Cancer Research UK to discover exactly how microbes in the body lead to bowel cancer. Part of the funding is earmarked for finding new ways to eliminate the prime suspects $-F$. nucleatum, $B$. fragilis and $E$. coli - without disturbing the entire microbial ecosystem inside the gut.

In 2017, Bullman, who was working in Meyerson's lab at the time, showed that the antibiotic metronidazole could slow cancer growth in mice with Fusobacterium-positive tumours ${ }^{7}$. But the antibiotic also targets a range of beneficial microbes in the intestines. "What we really need," says Bullman, "are more targeted approaches." A drug specific to Fusobacterium could do the trick. Or a vaccine. Or perhaps a phage therapy that takes advantage of bacteria-infecting viruses to precisely eliminate the nefarious microbe.

\section{Targeted attack}

At the 2019 International Cancer Immunotherapy Conference in Paris, scientists from microbiome therapeutic company BiomX in Ness Ziona, Israel, reported the discovery of several Fusobacterium-targeted phages that, when injected into the bloodstream of mice, could successfully invade bacteria nestled inside implanted tumours. According to chief executive Jonathan Solomon, BiomX is harnessing synthetic biology to turn the phages into programmable bacterial assassins that could also deliver a therapeutic payload that helps to recruit tumour-fighting immune cells to the site of attack.

Alternatively, predatory bacteria called Bdellovibrio could be deployed in the fight against cancer-causing microbes - and microbiologist Emma Allen-Vercoe from the University of Guelph, Canada, didn't need to look far to discover one that could destroy Fusobacterium. Her team dug up a patch of clover from the lawn in front of the university's clock tower. Among the germ-eating microbes they found, "some are real champions at killing fusobacteria", she says.

Despite these efforts, most specialists acknowledge that it remains to be shown definitively whether the human microbiome has a causal role in cancer. It could be that some bacteria are merely opportunistic invaders of tumours once cancer has already taken hold.

"We're lacking solid direct evidence in the form of longitudinal cohort studies that the microbiome causes cancer," says Alasdair Scott, a colorectal surgeon at Imperial College London. He is one of the architects of the International Cancer Microbiome Consortium, a global body that aims to establish expert consensus on the role of the microbiome in oncology. Last year, the group wrote a policy paper calling on researchers to address the question of causation versus association ${ }^{8}$.

\section{"Every day now there seems to be some new microbe associated with cancer."}

Prospective cohort studies take years, however, and new treatment options for cancer are needed now. "You can't wait until everything is completely known," Holt says. Clinicians are therefore moving ahead with testing microbiome modulation, especially in patients receiving checkpoint-inhibitor drugs designed to rev up the body's antitumour immune response.

At the 2019 meeting of the American Association for Cancer Research, two research groups described promising work in people receiving immunotherapy for melanoma. In both cases, the tumours of people who initially did not benefit from the treatment shrank after receiving faecal matter from someone who did respond to the drugs.

Elsewhere, researchers have been administering poo-stuffed pills from healthy donors alongside immunotherapy, with similarly favourable results. And, given that the procedure is generally considered to be low risk, some clinicians are beginning to think about a future in which stool swaps become a standard add-on to immunotherapy for all patients, not just those who fare poorly on the immune-targeted drugs to begin with. "Everybody might be doing it right off the bat," says John Lenehan, an oncologist at the London Regional Cancer Program in Canada.

The safety of faecal transplants was called into question, however, when researchers last year described how contaminated stool left one man dead and another severely ill in experimental trials investigating the procedure for other applications ${ }^{9}$. 


\section{outlook}

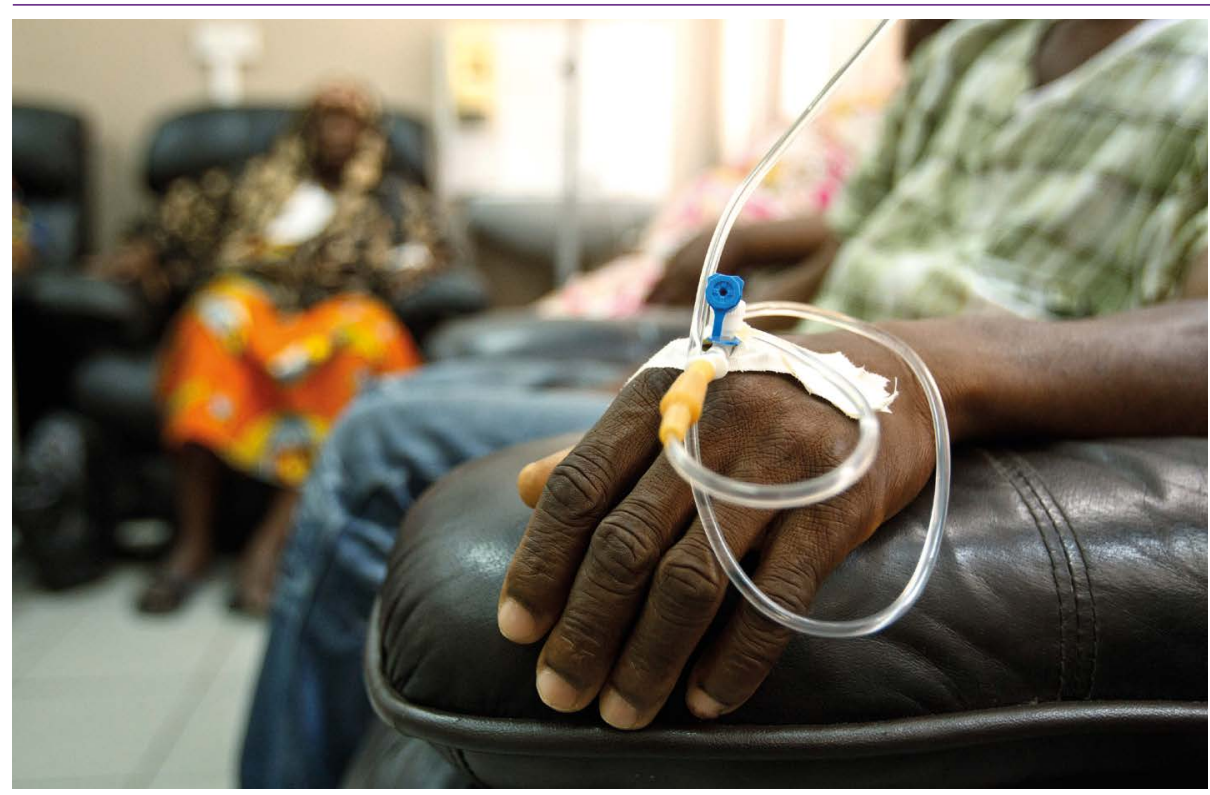

People with cancer might benefit from receiving microbial therapies alongside cancer drugs.

Physicians now test donated samples for the drug-resistant strain of $E$. coli that caused the infections. But fearing that a newly virulent microbe could slip through the screening process, some researchers are turning to defined, well-characterized and lab-grown formulations of microbes.

These blends of cultured strains are typically selected on the basis of observational human studies and mouse experiments that test which organisms most influence the response to immunotherapy. Wargo, for example, led one of a number of groups that described correlations between clinical responses to checkpoint inhibitors and the composition of the gut microbiome. Microbiomics company Seres Therapeutics in Cambridge, Massachusetts, took those findings, incorporated extra in-house data, and created a mix of strains from dozens of bacterial species, all in spore form. Researchers, including Wargo, have began testing the Seres product in people with advanced-stage melanoma.

Microbial therapeutics company Vedanta Biosciences, also in Cambridge, picked 11 strains for its bacterial cocktail by looking for microbes in human faeces that most potently elicited the desired immune responses in mice. A team including Vedanta's scientists showed how each strain in isolation could enhance antimicrobial or antitumour immunity in mouse models ${ }^{10}$. "However, assembled in certain consortia, they had a much larger effect," says study co-author and Vedanta chief executive Bernat Olle.

Some firms, including pharmaceutical company 4D Pharma in Leeds, UK, are paring down the therapeutic approach even further and administering single microbial strains with immune-stimulating effects. The company's scientists have described a strain of Enterococcus gallinarum, isolated from a healthy human gut, and its structural protein flagellin, which rouses the immune system by interacting with a receptor found on intestinal cells. The firm is now testing that strain in the clinic in people with lung, kidney, bladder and skin cancers, both as a therapy ahead of surgical removal of the tumours and in combination with a checkpoint inhibitor.

\section{Poo versus pills}

Bryan Coburn, an infectious-disease specialInstitute in Canada, points to several benefits of using rationally designed consortia of bacteria rather than relying on donor faecal material. "There are specific safety advantages, because we know exactly what's going in," says Coburn, who is clinically evaluating a multi-strain pill for cancer from NuBiyota, which is based in Pearl River, New York, and co-founded by Guelph's Allen-Vercoe. Prepared formulations are scalable and modifiable, Coburn says. Moreover, "we can assess things like potency, which you can't do easily with faecal transplants", he adds.

Microbial therapies might also need to be

"People are really embracing the idea and we're beginning that labour." ist at the Toronto General Hospital Research

"Peopleare really embracing
the idea and we're beginning
to see the early fruits of
that labour."

tailored to certain tumour types. For example, men with metastatic prostate tumours who responded to checkpoint inhibition have been found to have lower levels of a microbe called Akkermansia muciniphila in their stool than did men who did not respond. But the opposite is true of people with lung and kidney cancers - those with more A. muciniphila in their guts tended to fare better on the therapy.

Amy Moran, an immunologist at Oregon Health and Science University in Portland, thinks that the different treatments that people with different cancers receive might explain the discrepancy. "So many other types of drugs that these patients take might be impacting the composition of the microbiome," she says. She suspects that, for prostate cancer, the hormone therapies commonly used as first-line treatments might be to blame. Uncertain of which bacteria will be best in this context, Moran and her colleagues are starting by trying to boost immunotherapy response with complete faecal transplants.

Elsewhere in the oncology clinic, researchers are turning to microbiome therapeutics to manage some of the immune-related toxicity associated with checkpoint-blocking drugs. At the University of Texas MD Anderson Cancer Center, gastroenterologist Yinghong Wang is using faecal transplants to manage cases of immunotherapy-induced colitis. In 2018, she described how a woman with bladder cancer and a man with prostate cancer, both of whom developed side effects including bloody diarrhoea after receiving checkpoint inhibitors, saw their symptoms resolve after one or two transplants of stool from a healthy donor ${ }^{11}$. Wang has since treated another dozen or so people. "All of them seem to benefit from this treatment," she says.

None of Wang's patients had previously received faecal transplants to improve therapeutic responses. If they had, she suspects they would not have developed the side effects in the first place. Microbiome modulation might, therefore, offer a double benefit for people with cancer - enhancing response rates to other drugs while also guarding against the worst of their ill effects.

\section{Elie Dolgin is a science journalist in} Somerville, Massachusetts.

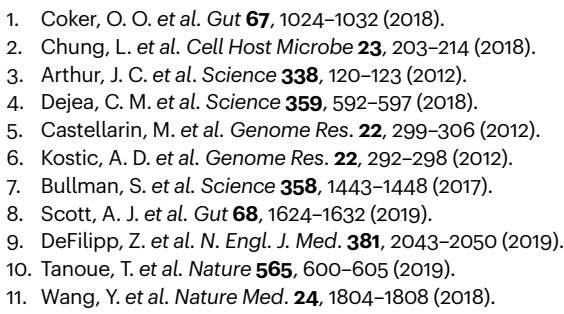

